\title{
Cálculo de Propriedades Termodinâmicas através da Equação de Estado Cúbica de Patel - Teja com os parâmetros estimados por Mota
}

Tânia Maria Galo ${ }^{1}$

Instituto Politécnico, IPRJ, UERJ, Nova Friburgo, RJ

Luiz Nélio H. Guedes de Oliveira ${ }^{2}$

Instituto Politécnico, IPRJ, UERJ, Nova Friburgo, RJ

Anny Resende Negreiros ${ }^{3}$

Instituto Federal do Espírito Santo - Cachoeiro de Itapemirim, ES, Brasil

Resumo. Este trabalho apresenta um estudo analítico sobre a acurácia dos parâmetros estimados por Mota [10] para a equação de estado de Patel-Teja no cálculo das propriedades termodinâmicas, volume de líquido saturado e entalpia de vaporização. Com o objetivo de avaliar o poder preditivo dessas propriedades termodinâmicas para tal equação foram utilizados dados experimentais, bem como os parâmetros da proposta original de Patel-Teja.

Palavras-chave. Patel-Teja, Mota, Volume de Líquido Saturado, Entalpia de Vaporização, Termodinâmica.

\section{Introdução}

Os cálculos de propriedades termodinâmicas são parte fundamental dos processos químicos nas indústrias [7]. Para que estes sejam feitos, são de grande relevância as avaliações de uma equação de estado (Equation of State, EOS). São reportadas na literatura três importantes equações de estado (cúbicas) para modelar processos de alta pressão, sendo elas a equação de Soave-RedlichKowng [16], a equação de Peng-Robinson [12] e a equação de Patel-Teja [11].

No cálculo da equação de estado são utilizados parâmetros estimados por métodos de otimização, que podem ser mono-objetivos $[2-4,9]$ ou multiobjetivos $[5,6,8]$. Recentemente, resultados demonstraram empiricamente certo conflito de modelos termodinâmicos equipados com os parâmetros encontrados via otimização mono-objetivo para calcular diferentes propriedades termodinâmicas [1,14]. Esses objetivos conflitantes em relação a acurácia de propriedades termodinâmicas influenciaram a utilização da otimização global multiobjetivo. Por exemplo na Ref. [6], os autores aplicaram a otimização multiobjetivo para estimação de parâmetros em cálculos de adsorção e equilíbrio de fases. Na Ref. [8], para ajustar os parâmetros de uma equação de estado aplicada em substâncias auto-associativas também foi aplicado com sucesso a otimização multiobjetivo. Da mesma forma, [5] utilizaram a otimização multiobjetivo para estimar parâmetros de um modelo multicomponente referente à simulação da destilação de cachaça em alambique, descrito em função das variáveis de controle de processo.

\footnotetext{
${ }^{1}$ taniamaria@iprj.uerj.br

2 nelio@iprj.uerj.br

3 anny.negreiros@ifes.edu.br
} 
Diante dos resultados obtidos com a otimização multiobjetivo, Mota [10] propôs, então, modificações nos parâmetros existentes na equação de estado de Patel-Teja. Com isso buscou aumentar o poder preditivo das propriedades termodinâmicas dessa equação e a confiança do emprego dessa EOS ao longo da região de saturação. Este trabalho, tem como objetivo averiguar analiticamente os resultados da equação de estado de Patel-Teja com os parâmetros obtidos por Mota [10]. Para isso, são realizados testes com as propriedades termodinâmicas dos resultados obtidos tanto por Patel-Teja quanto por Mota, comparando-os à dados experimentais.

\section{Formulação do Problema}

Considere a EOS de Patel-Teja [11] definida pela Eq. (1)

$$
P=\frac{R T}{V-b}-\frac{a[T]}{V(V+b)+c(V-b)}
$$

com os parâmetros

$$
a[T]=\Omega_{a} \frac{R^{2} T_{c}^{2}}{P_{c}} \alpha\left[T_{r}\right], \quad b=\Omega_{b} \frac{R T_{c}}{P_{c}} \quad \text { e } \quad c=\Omega_{c} \frac{R T_{c}}{P_{c}} .
$$

onde $R$ é a constante dos gases ideias, $V$ o volume molar, $T$ a temperatura, $T_{c}$ a temperatura crítica, $P_{c}$ a pressão crítica e $T_{r}=T / T_{c}$ a temperatura reduzida. Esses parâmetros são específicos para cada substância e $\alpha\left[T_{r}\right]$ é dada por:

$$
\alpha\left[T_{r}\right]=\left[1+m\left(1-\sqrt{T_{r}}\right)\right]^{2} .
$$

Os coeficientes adimensionais $\Omega_{a}, \Omega_{b}$ e $\Omega_{c}$ são parâmetros obtidos pelas restrições no ponto crítico [15]. Logo:

$$
\begin{gathered}
\Omega_{c}=1-3 \zeta_{c} \\
\Omega_{a}=3 \zeta_{c}^{2}+3\left(1-2 \zeta_{c}\right) \Omega_{b}+\Omega_{b}^{2}+1-3 \zeta_{c}
\end{gathered}
$$

onde $\Omega_{b}$ é a menor raiz positiva de

$$
\Omega_{b}^{3}+\left(2-3 \zeta_{c}\right) \Omega_{b}^{2}+3 \zeta_{c}^{2} \Omega_{b}-\zeta_{c}^{3}=0 .
$$

Na região crítica $0,9<\operatorname{Tr}<1,0, \zeta_{c}$ é definido por

$$
\zeta_{c}=\zeta_{c}-10\left(\zeta_{c}-Z_{c}\right)\left(T_{r}-0,9\right) \text {. }
$$

A Eq. (1) pode ser expressa na forma de polinômio cúbico para o fator de compressibilidade, $Z$, como

$$
Z^{3}-(1-C) Z^{2}+\left(A-B^{2}-2 B C-B-C\right) Z-\left(A B-B C-B^{2} C\right)=0
$$

onde $A, B$ e $C$ são dados por:

$$
A=\frac{a[T] P}{R^{2} T^{2}}, \quad B=\frac{b P}{R T} \quad \text { e } \quad C=\frac{c P}{R T} .
$$

Mota [10] determinou para 260 substâncias puras três novos pares de parâmetros para a equação de estado de Patel-Teja [11], um que privilegia o menor erro para a pressão de vapor, $P^{s a t}$, outro que diminui o desvio para o volume de líquido saturado, $V^{\text {sat }}$, e um terceiro intermediário a ambas 
as propriedades. Este trabalho avalia a predição das propriedades entalpia e volume de líquido com base nesses parâmetros estimados por Mota [10]. O volume de líquido é calculado mediante a solução da Eq. (10) com o fator de compressibilidade obtido para a menor raiz da Eq. (8) considerando os valores de temperatura e pressão de interesse.

$$
V=\frac{Z R T}{P}
$$

A entalpia de vaporização, $\Delta H^{v a p}$, pode ser determinada calculando-se a diferença entre a entalpia no estado de vapor e líquido,

$$
\Delta H^{\text {vap }}=\left(H-H^{0}\right)^{\text {vapor }}-\left(H-H^{0}\right)^{\text {liquido }},
$$

onde $H^{0}$ é a entalpia no estado ideal. A entalpia de vaporização é utilizada como base de comparações, pois os dados referentes à entalpia no estado de referência muitas vezes não são especificados.

A entalpia residual é relacionada a equação de estado pela equação:

$$
\left(H-H^{0}\right)=R T(Z-1)+\int_{\infty}^{V}\left[T\left(\frac{\partial P}{\partial T}\right)_{V}-P\right] d V
$$

Substituindo a Eq. (1) na Eq. (12), obtém-se

$$
\left(H-H^{0}\right)=R T(Z-1)-\left(T \frac{\partial a}{\partial T}-a\right)\left[\frac{1}{2 N} \ln \left(\frac{Z+M}{Z+Q}\right)\right],
$$

sendo $Z$ a solução da Eq. (8) para a temperatura e pressão desejada, onde os valores de $N, M$ e $Q$ são dados por:

$$
N=\sqrt{b c+\left(\frac{b+c}{2}\right)^{2}}, \quad M=\left(\frac{b+c}{2}-N\right) \frac{P}{R T} \quad \text { e } \quad Q=\left(\frac{b+c}{2}+N\right) \frac{P}{R T} .
$$

\section{Resultados}

As soluções analíticas para as propriedades definidas na Seção 2, foram implementadas no software de computação simbólica Wolfram Mathematica. Para os resultados a seguir utilizou-se as substâncias etano e metano conforme a Tabela 1.

Tabela 1: Propriedades físicas do etano e metano [13]

\begin{tabular}{ccccc}
\hline Substâncias & Fórmula & $T_{c} \mathrm{~K}$ & $P_{c} \mathrm{MPa}$ & $Z_{c}$ \\
\hline etano & $\mathrm{C}_{2} H_{6}$ & 305,32 & 4,872 & 0,279 \\
metano & $\mathrm{CH}_{4}$ & 190,564 & 4,599 & 0,286 \\
\hline
\end{tabular}

As propriedades calculas foram comparadas aos dados experimentais obtidos da literatura em mesmas condições de pressão e temperatura, através do cálculo do desvio médio relativo percentual:

$$
A A D(\%)=\left(\frac{100}{n}\right) \sum_{i=1}^{n} \frac{\left|\exp _{i}-\operatorname{calc}_{i}\right|}{\exp _{i}} .
$$

onde para um determinado dado $i$, calc $_{i}$ é o valor calculado, $\exp _{i}$ é o valor experimental e $n$ a quantidade de dados experimentais para a substância analisada. 
Tabela 2: Parâmetros $\zeta_{c}$ e $m$

\begin{tabular}{|c|c|c|c|c|c|c|c|c|}
\hline \multirow[b]{2}{*}{ Substância } & \multicolumn{2}{|c|}{$V^{\text {sat }}[10]$} & \multicolumn{2}{|c|}{ Intermediário [10] } & \multicolumn{2}{|c|}{$P^{s a t}[10]$} & \multicolumn{2}{|c|}{$\mathrm{PT}[11]$} \\
\hline & $m$ & $\zeta_{c}$ & $m$ & $\zeta_{c}$ & $m$ & $\zeta_{c}$ & $m$ & $\zeta_{c}$ \\
\hline etano & 0,586064 & 0,323257 & 0,572864 & 0,319565 & 0,557225 & 0,315281 & 0,561567 & 0,317 \\
\hline metano & 0,467387 & 0,327666 & 0,431710 & 0,318383 & 0,391338 & 0,306705 & 0,455336 & 0,324 \\
\hline
\end{tabular}

A Tabela 2 apresenta os parâmetros estimados $\zeta_{c}$ e $m$ para as substâncias etano e metano conforme os trabalhos de Mota [10] e de Patel-Teja [11].

As Tabelas 3 e 4 mostram os resultados dos desvios médios relativos percentual calculados a partir da Eq. (15). Observa-se para o volume de líquido saturado, que os parâmetros $V^{\text {sat }}$ propostos por Mota [10] são os que melhor predizem essa propriedade na região de saturação, faixa de temperatura $T_{r}$.

Tabela 3: $A A D \%$ Volume de líquido saturado

\begin{tabular}{ccccccc}
\hline Substâncias & $n$ & $T_{r}$ & $V^{\text {sat }}$ & Intermediário & $P^{\text {sat }}$ & PT \\
\hline etano & 17 & $0,45-0,99$ & $\mathbf{1 , 7 8 5 0 9}$ & 2,04588 & 2,96104 & 2,51293 \\
metano & 18 & $0,52-0,98$ & $\mathbf{2 , 4 6 6 2 4}$ & 3,63584 & 7,99026 & 2,63447 \\
\hline
\end{tabular}

Tabela 4: AAD \% Entalpia de vaporização

\begin{tabular}{ccccccc}
\hline Substâncias & $n$ & $T_{r}$ & $V^{\text {sat }}$ & Intermediário & $P^{\text {sat }}$ & $\mathrm{PT}$ \\
\hline etano & 17 & $0,45-0,99$ & $\mathbf{0 , 7 2 9 7 2 9}$ & 0,75149 & 0,798935 & 0,756696 \\
metano & 18 & $0,52-0,98$ & 1,19244 & 1,04176 & $\mathbf{0 , 9 8 4 7 1 9}$ & 1,20229 \\
\hline
\end{tabular}

As Figuras 1a-b mostram o comportamento das curvas obtidas para o volume de líquido saturado calculados com os parâmetros $\zeta_{c}$ e $m$. Pode-se observar uma boa concordância entre a curva dos dados experimentais e os valores estimados.

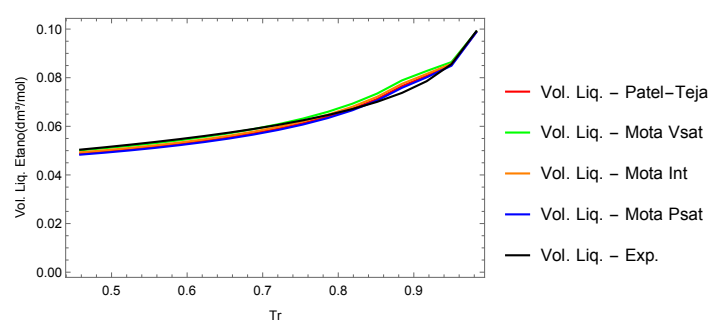

(a) Etano

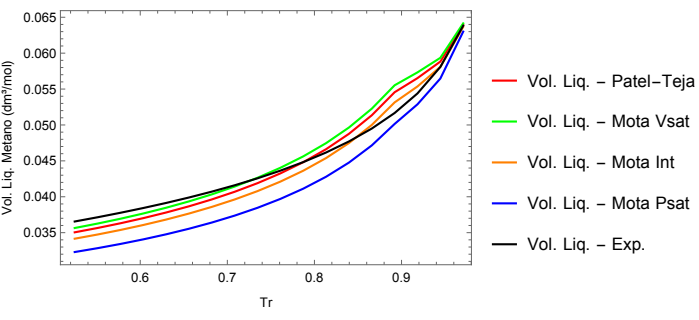

(b) Metano

Figura 1: Volume de líquido saturado.

Da mesma forma, as Figuras 2a-b mostram o comportamento das curvas obtidas para a entalpia de vaporização. Em ambos os casos, também observa-se a convergência entre as calculadas e os dados experimentais. 


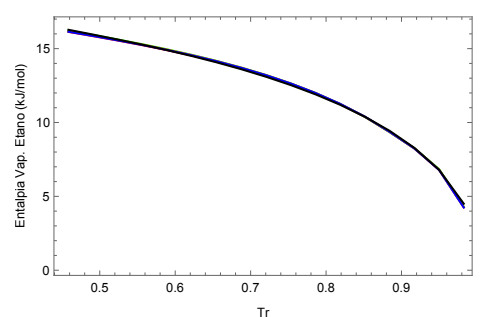

(a) Etano

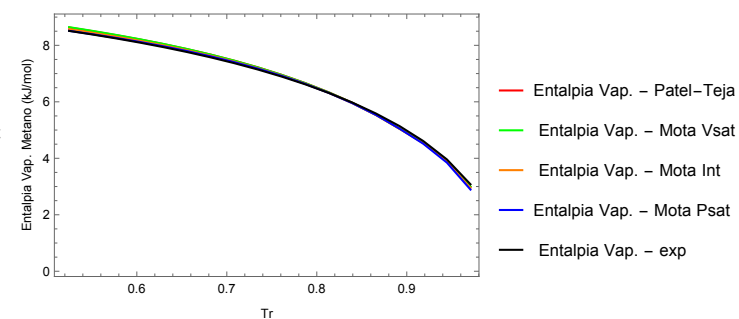

(b) Metano

Figura 2: Entalpia de vaporização.

A diferença entre os resultados experimentais e os calculados, Figuras 3a-b, mostram que o parâmetro $V^{\text {sat }}$ apresenta erros menores em regiões mais distantes da temperatura crítica.

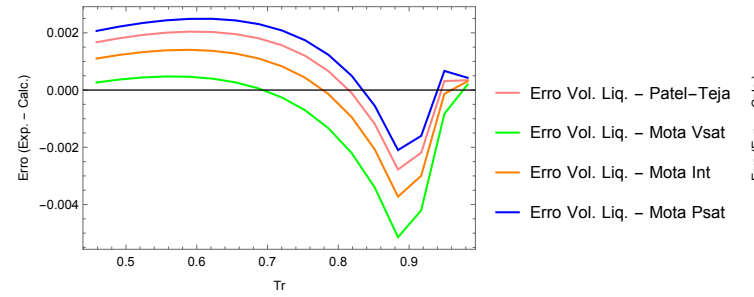

(a) Erro etano

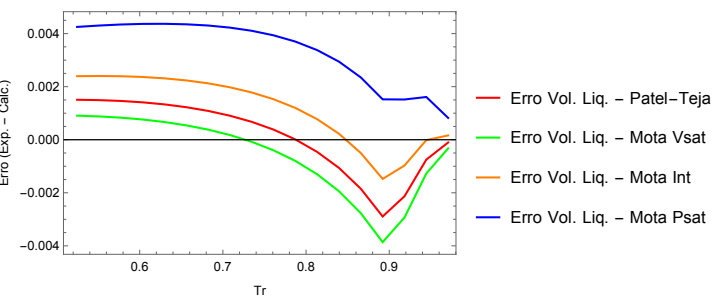

(b) Erro metano

Figura 3: Erro volume de líquido saturado.

No caso da entalpia de vaporização, Figuras 4a-b, observa-se que em regiões mais distantes da crítica o parâmetro $P^{\text {sat }}$ apresenta erros menores para o metano.

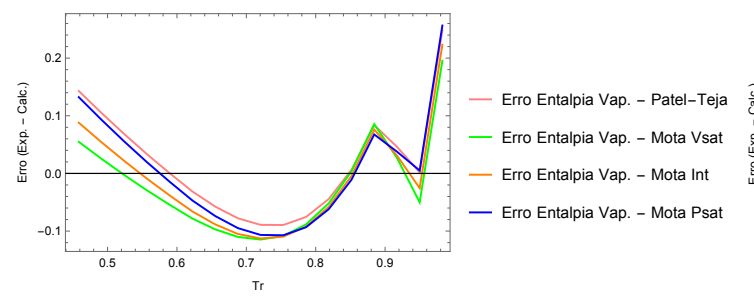

(a) Erro etano

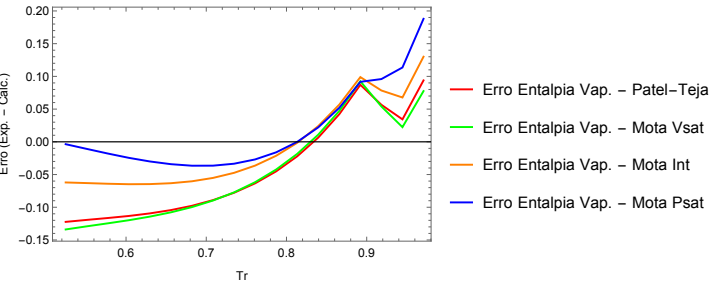

(b) Erro metano

Figura 4: Erro entalpia de vaporização.

\section{Conclusões}

Os resultados obtidos neste trabalho, evidenciam a acúracia dos parâmetros estimados por Mota [10] na predição de propriedades termodinâmicas saturadas de substâncias puras. Para essas conclusões foram analisados os resultados do volume de líquido saturado e da entalpia de vaporização válidos dentro de uma faixa de temperatura reduzida. Em comparação com a proposta original de Patel-Teja [11] e com os dados experimentais, a proposta de Mota [10] mostrou-se eficiente e capaz de aprimorar ainda mais a aplicação dessa equação. 


\section{Agradecimentos}

O presente trabalho foi realizado com apoio da Coordenação de Aperfeiçoamento de Pessoal de Nível Superior - Brasil (CAPES) - Código de Financiamento 001.

\section{Referências}

[1] Abdollaht-Demneh, F. et al. Comparison of the prediction power of 23 generalized equations of state: Part I. Saturated thermodynamic properties of 102 pure substances. Fluid phase equilibria, Elsevier, v. 288, n. 1-2, pages 67-82, 2010.

[2] Forero, L. A.; Velásquez, J. A. A method to estimate the Patel- Teja equation of state constants. Journal of Chemical \& Engineering Data, ACS Publications, v. 55, n. 11, pages 50945100, 2010.

[3] Forero, L.; Velásquez, J. The Patel - Teja and the Peng - Robinson EoSs performance when Soave alpha function is replaced by an exponential function. Fluid Phase Equilibria, Elsevier, v. 332, pages 55-76, 2012.

[4] Haghtalab, A. et al. A new three-parameter cubic equation of state for calculation physical properties and vapor-liquid equilibria. Fluid Phase Equilibria, Elsevier, v. 293, n. 2, pages 209-218, 2010.

[5] Junior, A. M. S. et al. A new pot still distillation model approach with parameter estimation by multi-objective optimization. Computers \& Chemical Engineering, Elsevier, pages 106-570, 2019.

[6] Kundu, P. K. et al. Genetic algorithm for multi-parameter estimation in sorption and phase equilibria problems. Chemical Engineering Communications, Taylor \& Francis, v. 205, n. 3, pages 338-349, 2018.

[7] Mak, P. C.-N. Thermodynamic properties from cubic equations of state. Tese (Doutorado), University of British Columbia, 1988.

[8] Menegazzo, T. A. et al. Application of an equation of state incorporating association to alcohols up to decanol. Fluid Phase Equilibria, Elsevier, v. 482, pages 24-37, 2019.

[9] Mohsen-Nia, M.; Modarress, H.; Mansoori, G. A cubic hard-core equation of state. Fluid phase equilibria, Elsevier, v. 206, n. 1-2, pages 27-39, 2003.

[10] Mota, B. T. Estimação de parâmetros de modelos termodinâmicos via otimização multiobjetivo. Tese (Doutorado), UERJ, 2019.

[11] Patel, N. C.; Teja, A. S. A new cubic equation of state for fluids and fluid mixtures. Chemical Engineering Science, Elsevier, v. 37, n. 3, pages 463-473, 1982.

[12] Peng, D.-Y.; Robison, D. B. A new two-constant equation of state. Industrial \& Engineering Chemistry Fundamentals, ACS Publications, v. 15, n. 1, pages 59-64, 1976.

[13] Perry, R. H., Green, D. W., Maloney, J. O. (1997). Perry's handbook of chemical engineering. Perry's Handbook of Chemical Engineering. 
[14] Punnapala, S.; Vargas, F. M.; Elkamel, A. Parameter Estimation in Phase Equilibria Calculations Using Multi-Objective Evolutionary Algorithms. Multi-Objective Optimization in Chemical Engineering: Developments and Applications, Wiley Online Library, pages 247-265, 2013.

[15] Smith, J. M., et al. Introdução à termodinâmica da engenharia química. $7^{\mathrm{a}}$ edição. LTC, Rio de Janeiro, v. 200, 2007.

[16] Soave, G. Equilibrium constants from a modified Redlich-Kwong equation of state. Chemical engineering science, Elsevier, v. 27, n. 6, pages 1197-1203, 1972. 